\title{
Differential Dynamic Microscopy: Probing wave vector dependent dynamics with a microscope
}

\author{
Roberto Cerbino ${ }^{(1,2)} * *$ and Veronique Trappe ${ }^{(1)}$ \\ (1) Department of Physics, University of Fribourg, \\ Chemin du Musée 3, CH-1700, Fribourg, Switzerland and \\ ${ }^{(2)}$ Department of Chemistry, Biochemistry and Medical Biotechnologies, \\ Universitá degli Studi di Milano, I-20133 Milano, Italy
}

\begin{abstract}
We demonstrate the use of an ordinary white-light microscope for the study of the q-dependent dynamics of colloidal dispersions. Time series of digital video images are acquired in bright field with a fast camera and image differences are Fourier-analyzed as a function of the time delay between them. This allows for the characterization of the particle dynamics independent on whether they can be resolved individually or not. The characteristic times are measured in a wide range of wavevectors and the results are found to be in good agreement with the theoretically expected values for Brownian motion in a viscous medium.
\end{abstract}


Microscopy and light scattering are widely used in physics, chemistry, biology and medical laboratories to access information on the structure and dynamics of mesoscopic systems. While microscopy gives direct access to real space images, scattering techniques work in reciprocal space, where information on the structure and dynamics of the system is obtained respectively from the angular and time dependence of the scattered light intensity [1]. These two complementary techniques have in general very different experimental requirements. White light sources are usual choices in microscopy, while a certain degree of coherence of the illuminating beam is required in scattering experiments; this is usually achieved by using a laser. In the past many attempts have been made to build a scattering apparatus based on a microscope; all of these attempts involved the use of a laser as an illumination source (see for example Refs. [2 6] and references therein). In some cases special care was taken to ensure the capability to perform microscopy and scattering experiments simultaneously, thereby allowing for a powerful combination of the complementary information obtained by both techniques [2, 3]. More recently, microscope-based laser Dynamic Light Scattering (DLS) experiments have been developed to study the dynamic properties of samples of biological interest, such as living macrophage and red blood cells [5, 6]. In practice, due to the intrinsic difficulties in building such instruments, the use of such techniques has been restricted to those laboratories, where a sufficient expertise in the realization of optical instrumentation was at hand.

In this letter, we present a conceptual scheme to interpret and analyze microscopy images that are obtained from samples containing moving entities. This technique, which we term Differential Dynamic Microscopy (DDM), does not entail any special experimental requirements, being based on the use of a standard light microscope with a normal illumination source and a digital video camera. By using the tools of Fourier Optics [7] we provide the means to access information about the sample dynamics that are equivalent to the one obtained in multi-angle dynamic light scattering (DLS) experiments [8]. We test DDM by analyzing time sequences of microscopy images obtained with an aqueous dispersion of colloidal particles with diameter $73 \mathrm{~nm}$, well below the resolution limit of the microscope. Additional tests are performed with larger particles (diameter $420 \mathrm{~nm}$ ). Our results are in good agreement with the predictions from the theory of Brownian motion in a viscous medium, thereby validating the proposed analysis. We believe that DDM is an interesting complement to the well established DLS [9] and microscope video tracking [10] techniques. 
Our experimental setup simply consists in an inverted microscope (LEICA DM IRB) equipped with a complementary metal-oxide-semiconductor (CMOS) camera (IDT X-Stream $\mathrm{XS}-3,1280 \times 1024$ pixels, pixel size $=12 \mu \mathrm{m})$. No modifications are made to the microscope. The sample is illuminated by focusing white light on the sample with a condenser lens (numerical aperture 0.9); for detection we use a standard objective (magnification $M=63 \times$, numerical aperture $N A=0.7)$. The effective pixel size, accounting for the magnification $M$, is then given by $d_{p i x}=0.19 \mu m$, value smaller than the microscope resolution limit imposed by diffraction. A capillary tube with rectangular section (Vitrocom, Inc.) is filled with an aqueous dispersion of polystyrene spheres of diameter $73 \mathrm{~nm}$ (Duke Scientific, part number $3070 \mathrm{~A})$. The concentration is $1 \%$ by weight fraction, sufficiently low to neglect the effect of interactions between individual spheres. The thickness of the capillary tube along the microscope optical axis is $100 \mu \mathrm{m}$ and a region at the center of the capillary tube is imaged onto the camera sensor.

A sequence of $N=1000$ images is acquired with a sampling rate of 100 samples/s and with an exposure time of $1 \mathrm{~ms}$. In Fig.1(a) we show a typical image: the image appears as a bright background with some dark spots. These dark spots are dust particles located mainly on the protective glass of the camera sensor. The signal due to the particles is barely visible as its contribution is small compared to the large background signal. However, the background is a static contribution, while the Brownian motion of the particles causes an incessant rearrangement of their configuration in space. By subtracting two images grabbed at different times we can eliminate the static contributions in our image and bring the displaced particles to light. Examples of difference images obtained by this subtraction procedure are shown in Fig.1(b) and 1(c); (b) and (c) are respectively the difference images obtained by subtracting two images separated in time by $\Delta t=10 \mathrm{~ms}$ and $\triangle t=200 \mathrm{~ms}$. Clearly, the grainy appearance of the difference images increases with increasing time delay $\triangle t$, revealing the increasing displacement of the particles.

We can quantify this increase by calculating the spatial variance

$$
\sigma^{2}(\triangle t)=\int|D(x, y ; \triangle t)|^{2} d x d y
$$

of the difference signal $D(x, y ; \triangle t)=I(x, y ; \triangle t)-I(x, y ; 0)$ as a function of $\triangle t$. Here, $I(x, y ; t)$ is the intensity obtained in the sensor plane $(x, y)$ at the time $t$, where we define the acquisition time of the first image to be $t=0 \mathrm{~s}$. The actual choice of the reference 

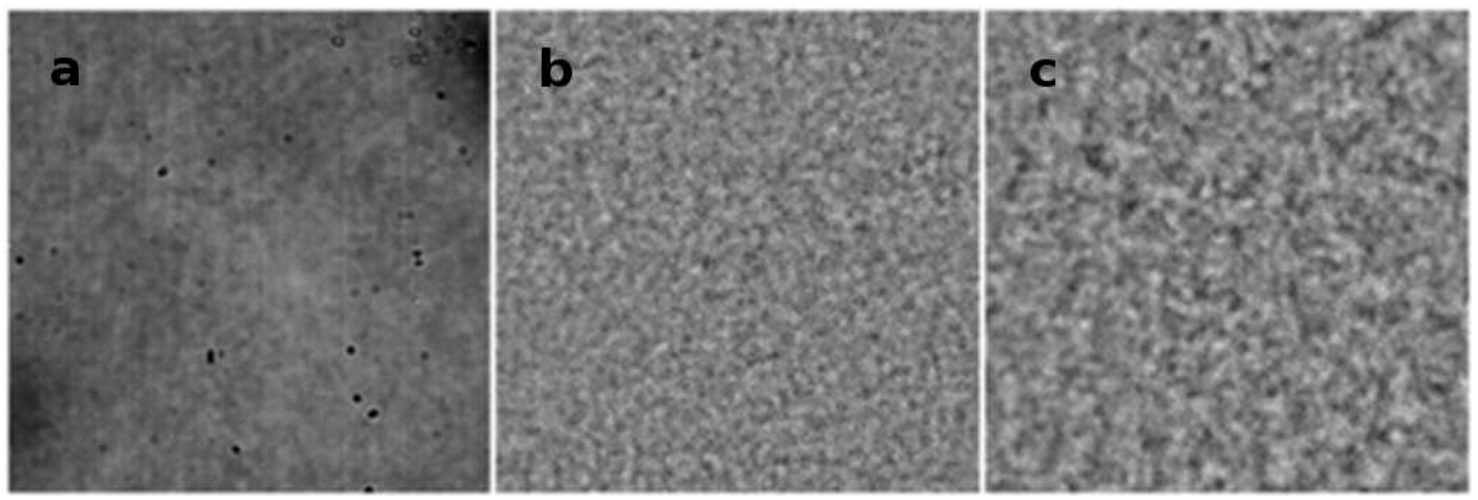

FIG. 1: (a) Microscope image of an aqueous dispersion of $73 \mathrm{~nm}$ particles at a weight fraction of $1 \%$. The particles are below the resolution of the microscope and the signal generated by them is very small. (b) Result of the subtraction of two images taken $10 \mathrm{~ms}$ apart in time. The signal due to the particles is now visible. (c) Result of the subtraction of two images $200 \mathrm{~ms}$ apart in time. The signal has increased. The size of each panel corresponds to $47 \mu \mathrm{m}$ in the sample.

image is not crucial in the case of Brownian motion, since the statistical properties of the investigated dynamics do not change in time. This can be appreciated in the movie clips shown in [11], where the time delay between two subtracted images is kept constant at $\triangle t=200 \mathrm{~ms}$. Consequently, for every $\triangle t$ we calculate the variance $\sigma^{2}(\triangle t)$ by averaging over many statistical realizations of the signal, typically 100, to improve the statistical accuracy. As shown in Fig.2, the variance $\sigma^{2}(\Delta t)$, increases very rapidly with $\triangle t$ and attains a steady state plateau at long times, when all the particles have moved far away from their initial position, such that the two images used to obtain the difference image are statistically uncorrelated. This dependence of the variance on $\Delta t$ clearly indicates that difference images can be used to monitor the particle dynamics. The question then arises whether it is possible to extract quantitative information on the dynamics of the system from the difference images. The answer is yes, but its justification requires the use of two steps.

The first one is to consider that the variance, defined in Eq.1 can be calculated in the Fourier space as well. If we define the 2D Fourier transform of $D(x, y ; \triangle t)$ as $F_{D}\left(u_{x}, u_{y} ; \triangle t\right)=\int D(x, y ; \triangle t) \exp \left[-i 2 \pi\left(u_{x} x+u_{y} y\right)\right] d x d y$, the use of the Parseval theorem [7] guarantees that

$$
\sigma^{2}(\triangle t)=\int\left|F_{D}\left(u_{x}, u_{y} ; \triangle t\right)\right|^{2} d u_{x} d u_{y}
$$




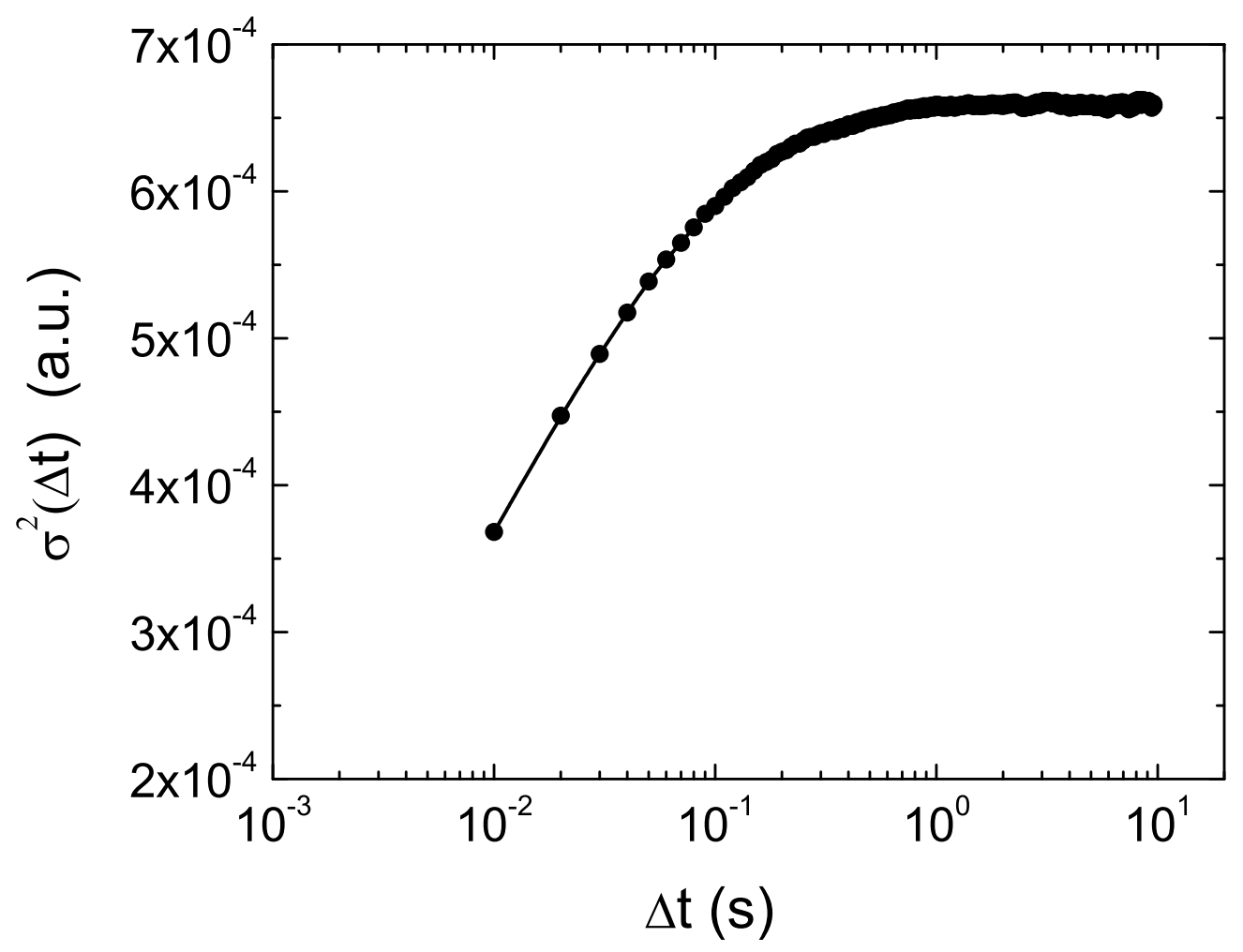

FIG. 2: The particles rearrangement due to their Brownian motion causes an increase of the variance $\sigma^{2}(\triangle t)$ of the difference signal $D(x, y ; \triangle t)$. With increasing time delay the two subtracted images become progressively uncorrelated, such that $\sigma^{2}(\triangle t)$ saturates at large $\triangle t$.

The equivalence of Eq, 1 and 2 shows that the total energy content of $D$ in real space is the same as the one of $F_{D}$ in Fourier space. However, in Fourier space it is possible to isolate every Fourier component and study the growth of its amplitude as a function of $\triangle t$.

To clarify whether this mode decomposition provides insight about the dynamics of the system we need to go through the second step. This step consists in realizing that a Fourier component of the microscope image is associated in a simple way to a scattering angle and in turn to a scattering wavevector. According to Abbe's theory of microscope image formation [7, 12], the object to be imaged on the camera sensor can be thought as the superposition of different Fourier components characterized by a spatial frequency $u_{o b j}$. Each frequency component of the object acts as a grating and diffracts light at an angle $\vartheta=\sin ^{-1}\left(\lambda u_{o b j}\right)$ with respect to the microscope optical axis, where $\lambda$ is the wavelength of light. On the camera sensor, the diffracted plane wave will produce a sinusoidal fringe pattern, characterized by a spatial frequency $u_{\text {det }}=\sin (\vartheta) / \lambda=u_{o b j}$, independent of the wavelength $\lambda$. This guarantees 


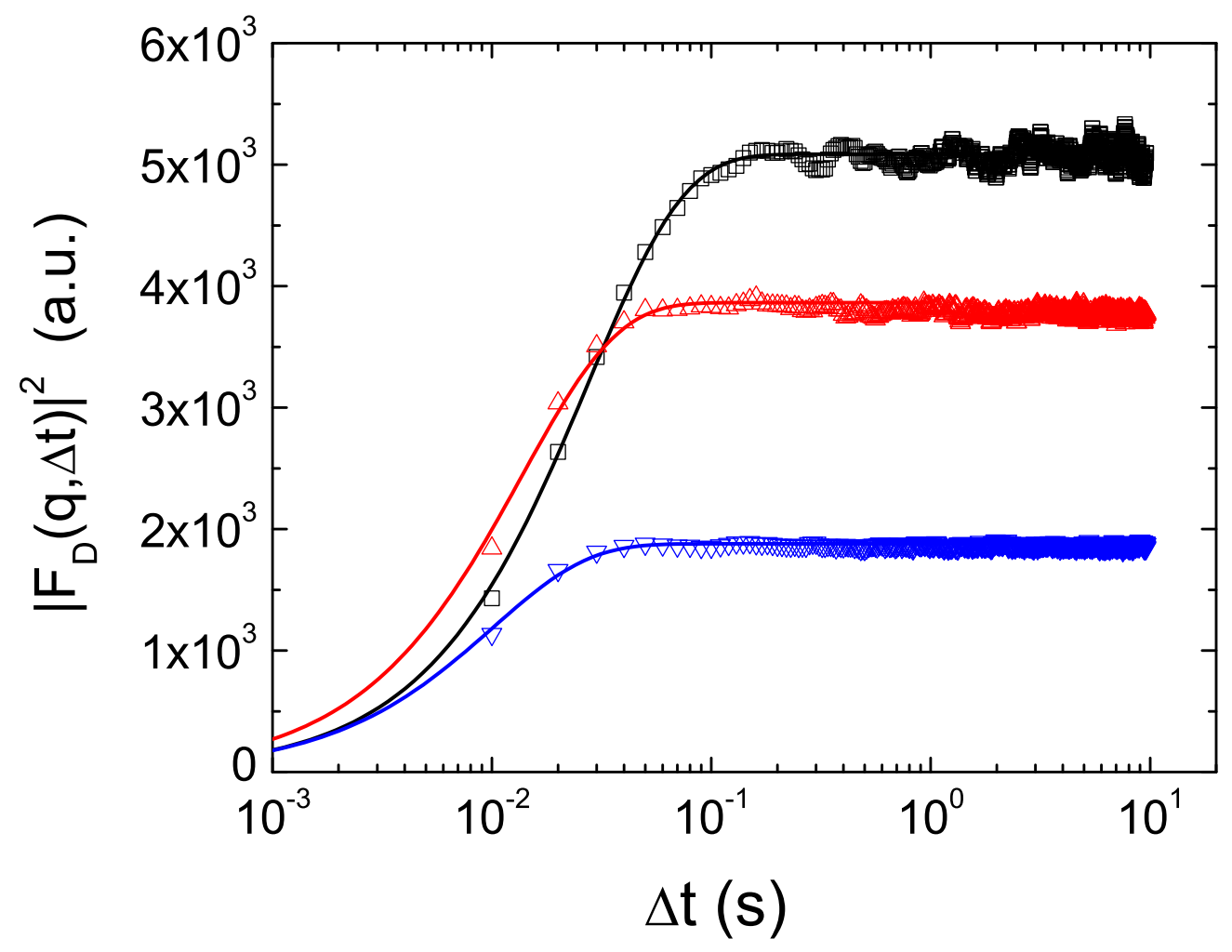

FIG. 3: Growth of $\left|F_{D}(q ; \Delta t)\right|^{2}$ with $\triangle t$ for three values of q: $q=2.6 \mu m^{-1}$ (black squares) , $q=3.9 \mu \mathrm{m}^{-1}$ (red up-triangles) and $q=4.5 \mu \mathrm{m}^{-1}$ (blue down-triangles). The continuous lines are fits of the data to $\mathrm{Eq} 3$.

that we can associate in a unique way, a spatial frequency $u_{\text {det }}$ on the detector to a scattering angle $\vartheta$.

To investigate the angular dependent dynamics of our system we thus use the Fourier power spectrum $\left|F_{D}\left(u_{x}, u_{y} ; \triangle t\right)\right|^{2}$, which we simply calculate by applying a Fast Fourier Transform [13] to the difference images. For isotropic samples, like the one investigated, the expectation value of $\left|F_{D}\left(u_{x}, u_{y} ; \Delta t\right)\right|^{2}$ is rotationally invariant in the $\left(u_{x}, u_{y}\right)$ plane. This allows us to perform azimuthal averages and to treat the one-dimensional power spectrum $\left|F_{D}(u ; \triangle t)\right|^{2}$, where $u=\sqrt{u_{x}^{2}+u_{y}^{2}}$. For easy comparison with scattering experiments we use wavevectors $q=2 \pi u$ instead of spatial frequencies $u$. Processing the data at several $\Delta t$ enables us to report the one-dimensional power spectrum $\left|F_{D}(q ; \Delta t)\right|^{2}$ as a function of the time delay, as shown for three values of $q\left(q=2.6 \mu m^{-1}, q=3.9 \mu m^{-1}\right.$ and $\left.q=4.5 \mu m^{-1}\right)$ in Fig.3. As for the variance $\sigma^{2}(\triangle t)$ we find that $\left|F_{D}(q ; \triangle t)\right|^{2}$ increases rapidly with $\triangle t$ to then saturate at longer time delays. The cross-over to the plateau value systematically shifts 
to smaller $\triangle t$ as the wavevector $q$ increases, denoting a $q$-dependence in the characteristic time of the system.

For a quantitative description of this behavior we consider that fluctuations in the intensity of the original images are due to concentration fluctuations in the sample. For Brownian diffusion it is well known that every Fourier concentration mode decays exponentially in time, $\exp (-\triangle t / \tau(q))$, with a characteristic time $\tau(q)=1 / D_{m} q^{2}$, where $D_{m}$ is the mass diffusion coefficient of the particles [9]. For the analysis of the difference images it is then easy to show that

$$
\left|F_{D}(q ; \triangle t)\right|^{2}=A(q)[1-\exp (-\triangle t / \tau(q))]+B(q)
$$

This equation contains two main contributions: the term $B(q)$ is due to the power spectrum of the camera noise and is present even in absence of the particles. The term $A(q)[1-\exp (-\triangle t / \tau(q))]$ describes the contribution associated to the particles. A quantitative description of the term $A(q)$ requires the knowledge of the exact relationship between intensity and concentration fluctuations, which is beyond the scope of this paper. However once $q$ is fixed, we can treat $A(q)$ and $B(q)$ as simple adjusting parameters and extract the characteristic time $\tau(q)$ by simply considering the $\triangle t$-dependence of $\left|F_{D}(q ; \triangle t)\right|^{2}$. This is very similar to what has been done in Ref. [14] where an expression similar to Eq,3 has been proposed to analyze sequences of shadowgraph images for the characterization of nonequilibrium concentration fluctuations in a binary mixture.

Thus, we fit our data to Eq.3 to obtain $\tau(q)$, which we report in Fig.4 for $q$ ranging from $0.4 \mu^{-1}$ to $5 \mu^{-1}$ (black open circles). On the same graph the dotted black line denotes the expected behavior calculated by using the formula $\tau(q)=1 / D_{m} q^{2}$, where $D_{m}$ is estimated using the Stokes-Einstein relation $D_{m}=k_{B} T /(3 \pi \eta d)=5.98 \mu \mathrm{m}^{2} / \mathrm{s} ; k_{B}$ is the Boltzmann constant, $T$ the absolute temperature, $\eta$ the solvent viscosity and $d$ the diameter of the particles. The experimental results are in good agreement with the theoretically expected values over more than one decade in $q$, showing that DDM is a reliable means to determine the dynamical properties of the sample. To our knowledge this result provides the first experimental evidence that white light, bright-field microscopy can be used to monitor the $q$-dependent dynamics of particles.

We also perform experiments by using particles with a diameter of $420 \mathrm{~nm}$, value comparable to the point spread function of the microscope. In Fig.5 we present two images of this sample separated in time by $10 \mathrm{~s}$. In contrast to the previous case the particles are 


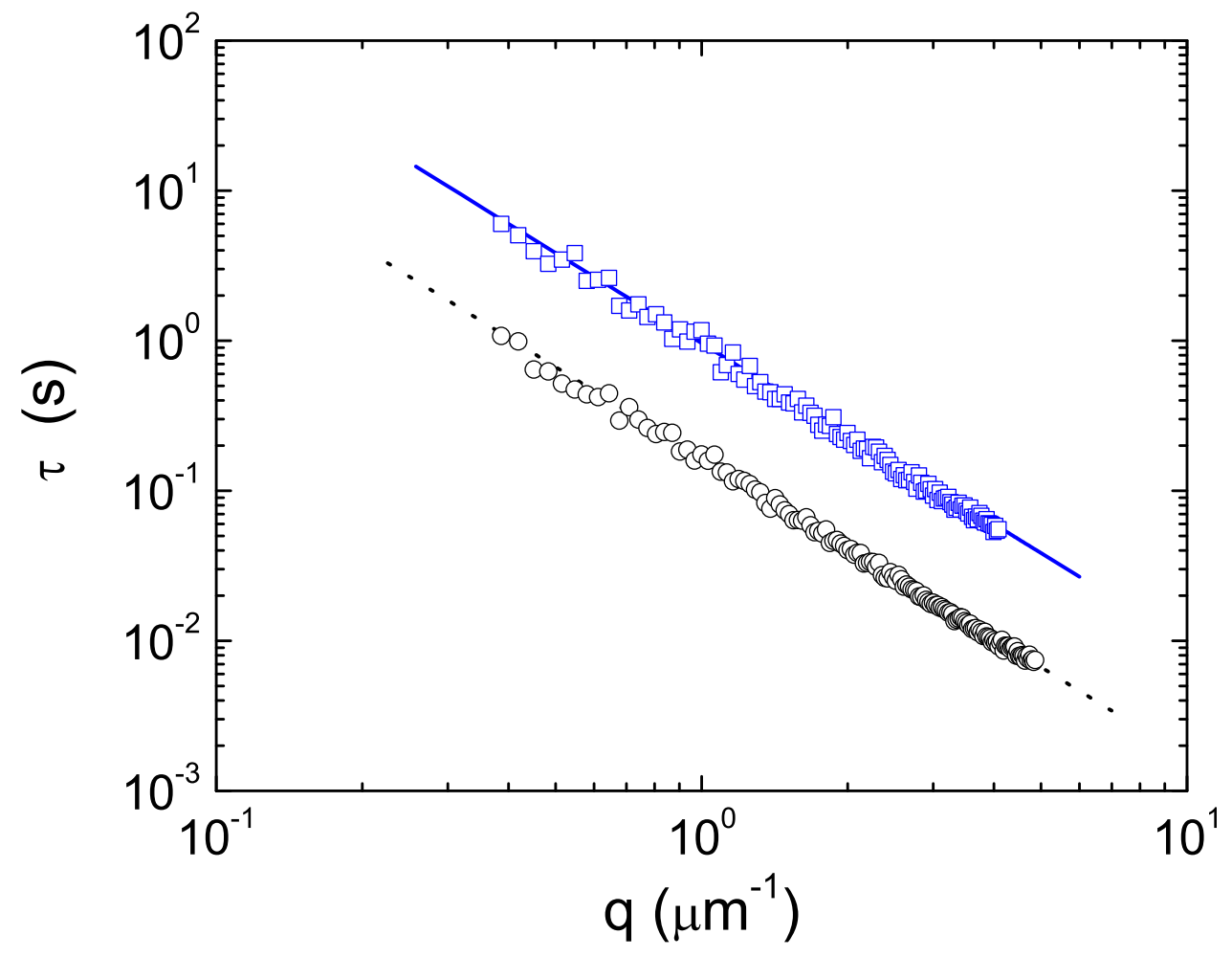

FIG. 4: Characteristic decay time $\tau$ versus the wavevector $q$ for the $73 \mathrm{~nm}$ (black circles) and the $420 \mathrm{~nm}$ (blue squares) particles. Fitting of the data gives respectively the values $D_{m}=6.2 \pm 0.3$ $\mu \mathrm{m}^{2} / \mathrm{s}$ and $D_{m}=1.1 \pm 0.2 \mu \mathrm{m}^{2} / \mathrm{s}$. The lines are theoretical predictions calculated by using the Stokes-Einstein relation with no adjustable parameters.

fairly visible, though the static signal due to dust along the optical path still represents a significant contribution. However, the subtraction procedure described above can still be applied to visualize the particles motion and to eliminate the static noise, as shown in the movie clip presented in [11]. The image sequence is analyzed as described above and the results are presented in Fig.4 as blue open squares. A good agreement with the theoretically expected behavior $\left(D_{m}=1.04 \mu \mathrm{m}^{2} / \mathrm{s}\right.$, continuous blue line) is also found in this case, demonstrating that DDM is capable of monitoring the dynamics of particles that can be either resolved individually or not. This suggests that DDM could be a valid complement to tracking techniques [10] for relatively big particles, with the advantage of being also applicable to concentrated systems, where tracking becomes difficult, and to fluctuating systems which are not composed by particles. Typical examples are membranes, interfaces or liquid crystals. For small particles DDM can be subsidiary to DLS experiments [9], because it can 

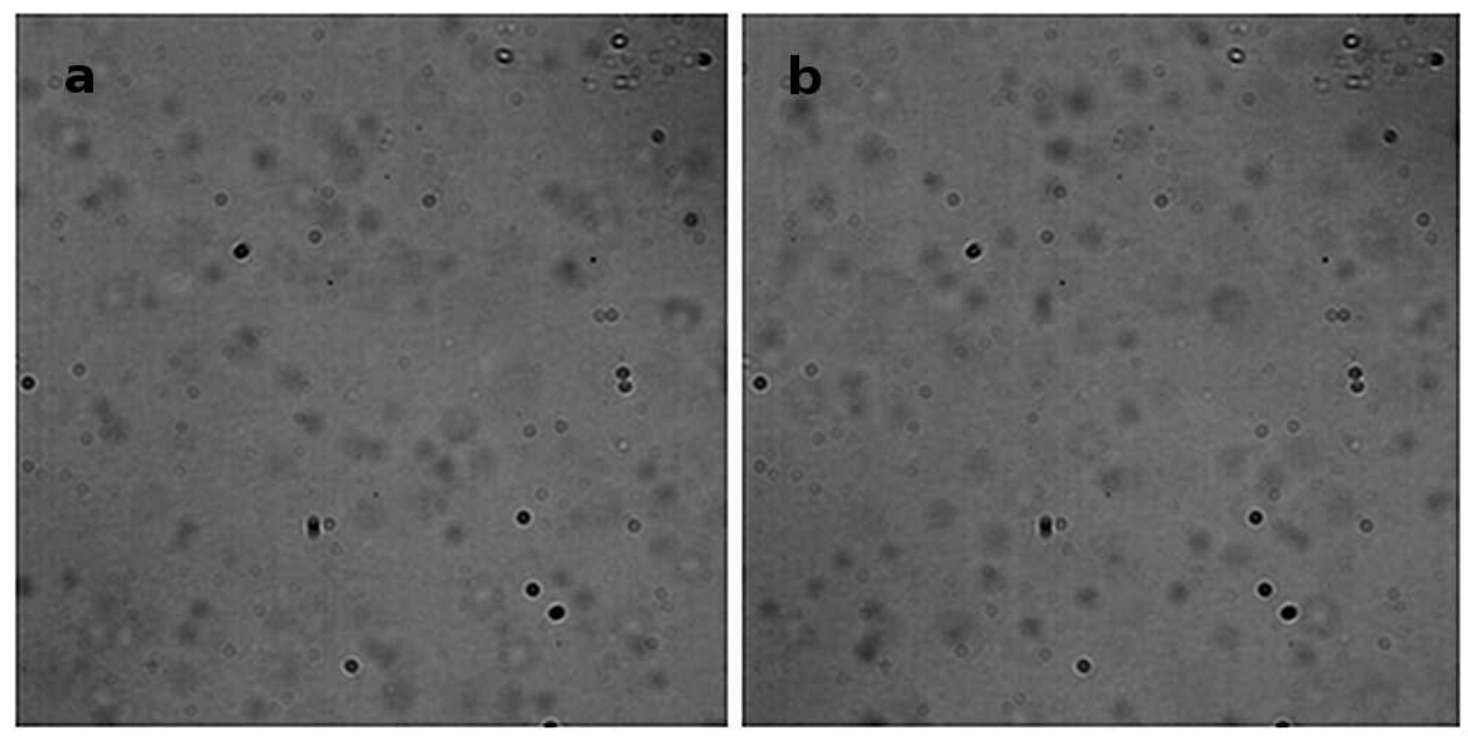

FIG. 5: Microscope images taken $10 \mathrm{~s}$ apart. The side of each panel corresponds to $47 \mu \mathrm{m}$ in the sample. The sample is a colloidal dispersion of $420 \mathrm{~nm}$ particles. The more visible objects are dust particles on the CMOS sensor which are also present in Fig.1(a), grabbed with another sample. The particles are the less visible objects that change position between image (a) and (b). The smearing effect due to the finite point spread function of the microscope is visible.

operate at small wavevectors where stray light makes the use of DLS notoriously difficult. The range of accessible time-scales is limited by the inverse of the acquisition frame rate on the lower side and by the overall duration of the experiment on the higher end.

A possible extension of this work could be the investigation of dynamically heterogeneous systems, where a space resolved investigation of the dynamics is of fundamental importance [15]. Our technique could also be applied to absorbing samples and to fluorescence microscopy, which is widely used for the investigation of biological samples [16]. Finally, we believe that DDM can be profitably used to perform microrheology experiments as pioneered in Ref. [17].

The authors thank M. Carpineti, F. Giavazzi and A. Vailati for stimulating discussions and for critical reading of the manuscript. T. Bellini, P. Cicuta, F. Croccolo, M. Giglio, R. Piazza, C. Takacs and D. Weitz are thanked for fruitful discussions. R.C. acknowledges financial support from the European Union (Marie Curie Intra-European Fellowship, contract EIF - 038772) and V.T. financial support from the Swiss National Science Foundation. 
* Electronic address: roberto.cerbino@unimi.it

[1] B. Chu, Laser Light Scattering: Basic Principles and Practice, (Dover, New York, 2007, 2nd ed)

[2] P. D. Kaplan, V. Trappe, and D. A. Weitz, Appl. Opt. 38, 4151 (1999).

[3] M. T. Valentine, A. K. Popp, P. D. Kaplan, and D. A. Weitz, Opt. Lett. 26, 890 (2001).

[4] R. Dzakpasu, and D. Axelrod, Biophys. J. 87, 1279 (2004).

[5] R. Dzakpasu, and D. Axelrod, Biophys. J. 87, 1288 (2004).

[6] M. S. Amin, Y. Park, N. Lue, R. R. Dasari,K. Badizadegan, M. S. Feld, and G. Popescu., Opt. Exp. 15, 17001 (2007).

[7] J. W. Goodman, Introduction to Fourier Optics, (Roberts\&Company, Englewood, 2005, 3rd ed).

[8] L. Cipelletti, and D. A. Weitz, Rev. Sci. Instrum. 70, 3214 (1999).

[9] B. J. Berne, R. Pecora, Dynamic Light Scattering: With Applications to Chemistry, Biology, and Physics, (Dover, New York, 2000).

[10] J. C. Crocker, and D. G. Grier, J. Colloid Interface Sci. 179, 298 (1996).

[11] See EPAPS Document No. [number will be inserted by publisher] for $50 \mathrm{~Hz}$ movie clips comparing the time evolution of the original images to the one of difference images with $\triangle t=200 \mathrm{~ms}$. 73nm.mpg and 420nm.mpg are for $73 \mathrm{~nm}$ and $420 \mathrm{~nm}$ correspondingly. For more information on EPAPS, see http://www.aip.org/pubservs/epaps.html

[12] H. Kohler, Opt. Acta. 28, 1691 (1981).

[13] R. N. Bracewell, The Fourier Transform and Its Application, (McGraw-Hill, New York, 1999, 3rd ed).

[14] F. Croccolo, D. Brogioli, A. Vailati, M. Giglio and D.S. Cannell, Ann. N.Y. Acad. Sci. 1077, 365 (2006).

[15] L. Cipelletti, and L. Ramos, J. Phys. : Condens. Matter 17, R253 (2005).

[16] J. W. Lichtman, and J. A. Conchello, Nat. Methods 2, 910 (2005).

[17] T. G. Mason, and D. A. Weitz, Phys. Rev. Lett. 74, 1250 (1995). 